Proc Indian Natn Sci Acad $\mathbf{8 1}$ No. 1 February 2015 Special Issue, pp. 28-35

(c) Printed in India.

DOI:10.16943/ptinsa/2015/v81i1/48047

\title{
On Direct Photon Production at RHIC and LHC-energies: A Theoretical Approach P GUPTAROY $^{*}$ \\ Department of Physics, Raghunathpur College, P.O.: Raghunathpur 723133, Dist.: Purulia (WB), India
}

(Received on 13 April 2014; Accepted on 10 June 2014)

\begin{abstract}
The Relativistic Heavy Ion Collider (RHIC) and the Large Hadron Collider (LHC) have recently studied different collisions at extremely high energies and produced a sizable amount of high-precision data. In the present work, we deal with the direct photon production phenomena in $p+p, d+A u A u+A u$ collisions at RHIC energy $\sqrt{s_{N N}}=200 \mathrm{GeV}$ and in $P b+P b$-collisions at LHC energy $\sqrt{s_{N N}}=2.76$ $\mathrm{TeV}$ on the basis of Sequential Chain Model (SCM). Comparisons of the model-based results with the measured data on some observables are generally found to be modestly satisfactory.
\end{abstract}

\section{Key Words : Relativistic Heavy Ion Collisions; Inclusive Production; Photon Production; Pion- Induced Nuclear Reactions; Quark-Gluon Plasma; Field Theory}

\section{Introduction}

The production of direct photon in high energy heavy ion collisions has been recognised as one of the important signals of the proposed Quark Gluon Plasma (QGP)-phase. The reasons are as follows: (i) By definition, direct photons are those photons which are not produced by the decays of either neutral pions or eta-mesons; (ii) Secondly, once produced they hardly ever interact and hence leave the system with their energy and momenta unaltered. But, till date, the exact nature of QGP-hadron phase transition is not known definitely (Nayak and Sinha, 2013). As the origin of the direct photon is somewhat different from that of thermal photon, it is open to various interpretations.

Here, in this work, we try to use an alternative model for the production of direct photon which is generally a non-standard type and is known as Sequential Chain Model (SCM). Our objectives are: (i) to interpret the latest data of direct photon production at RHIC and LHC, at energies $\sqrt{s_{N N}}=200 \mathrm{GeV}$ and

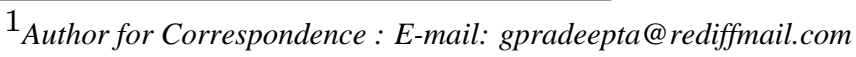


2.76 TeV respectively, with the help of the SCM and compare it with some other standard approaches; and (ii) to put this alternative approach for understanding the nucleonic structure and the reaction mechanism at high energy nuclear collisions.

The plan of this work is as follows. In next section we give a brief outline of the basic approach, the SCM. In the following section the results arrived at have been presented with figures. And in the last section we offer the final remarks and conclusions.

\section{The Outline of the Model}

The salient features and the important physical characteristics for the direct photon production in the light of the SCM valid for hadron-hadron collisions are as follow: according to this model, high energy hadronic interactions boil down essentially to the pion-pion interactions; as the protons are conceived in this model as $p=\left(\pi^{+} \pi^{0} \vartheta\right)$, where $\vartheta$ is a spectator particle needed for the dynamical generation of quantum numbers of the nucleons. The incident energetic $\pi$-meson emits a $\rho$-meson, the $\rho$-meson then emits a $\pi$-meson and is changed into a $\omega$-meson, the $\omega$-meson emits a photon, which is called the direct photon $\left(\gamma_{d}\right)$ form the viewpoint of the present model, and the $\pi$-meson is transformed once again into $\rho$-meson and the process of direct photon production continues (Guptaroy and Guptaroy, 2013; Guptaroy et al., 2008, 2003a,b).

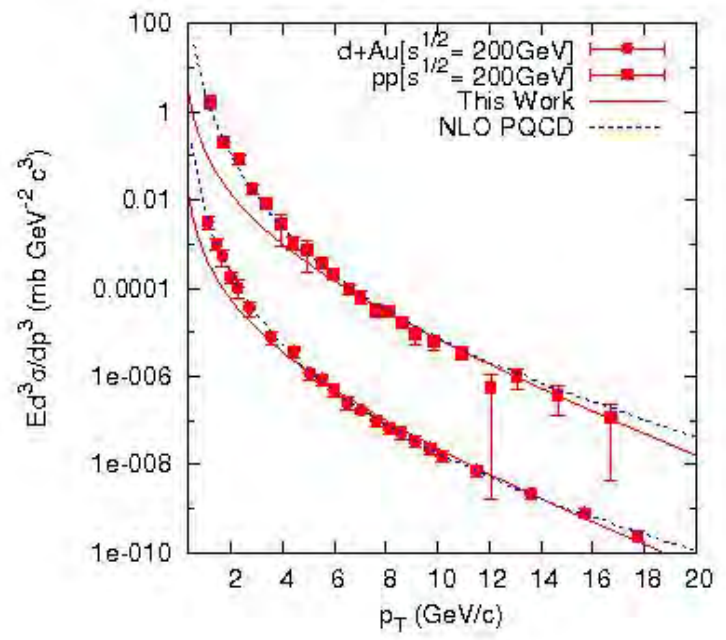

Fig. 1: Plot of the invariant cross-sections for direct photon production in proton-proton and deuterium-gold collisions at $\sqrt{s}_{N N}=200 \mathrm{GeV}$ as function of $p_{T}$. The data points are from (Wilde et al., 2013). The solid lines show the SCM-based results while the dotted lines represent NLO PQCD-based output

The basic expression for inclusive cross-section for production of direct photon in SCM is obtained for proton-proton collisions by using Feynman diagram techniques and infinite momentum frame approximation. The expressions for the reaction of the type $p+p \rightarrow Q+x$ were derived field theoretically (Guptaroy 
et al., 2003b). In order to study a nuclear interaction of the type $A+B \rightarrow Q+x$, where $A$ and $B$ are projectile and target nucleus respectively, and $Q$ is the detected particle which, in the present case, would be direct photon, the SCM has been adapted to a fly over model (FOM). The modification of the SCM or the FOM is constructed on the basis of the suggestions from Wong (Wong, 1994), the Glauber techniques and by using Wood-Saxon distributions (Gorenstein et al., 2002). The details of the physics regarding this model are given in Refs.(Guptaroy and Guptaroy, 2013; Guptaroy et al., 2008, 2003a,b). The inclusive cross-sections for direct photon production in different nuclear interactions of the types $A+B \rightarrow \gamma_{d}+x$ in the light of this modified SCM can then be written in the following generalised form as (Guptaroy and Guptaroy, 2013; Guptyaroy et al., 2008, 2003a,b):

$$
\left.E \frac{d^{3} \sigma}{d p^{3}}\right|_{A B \rightarrow \gamma_{d} x}=a_{\gamma_{d}} p_{T}-N_{R}^{\gamma_{d}} \exp \left(-\Delta^{\gamma_{d}} p_{T}\right)
$$

where $a_{\gamma_{d}}, N_{R}^{\gamma_{d}}$ and $\Delta^{\gamma_{d}}$ are the factors to be calculated under certain physical constraints.

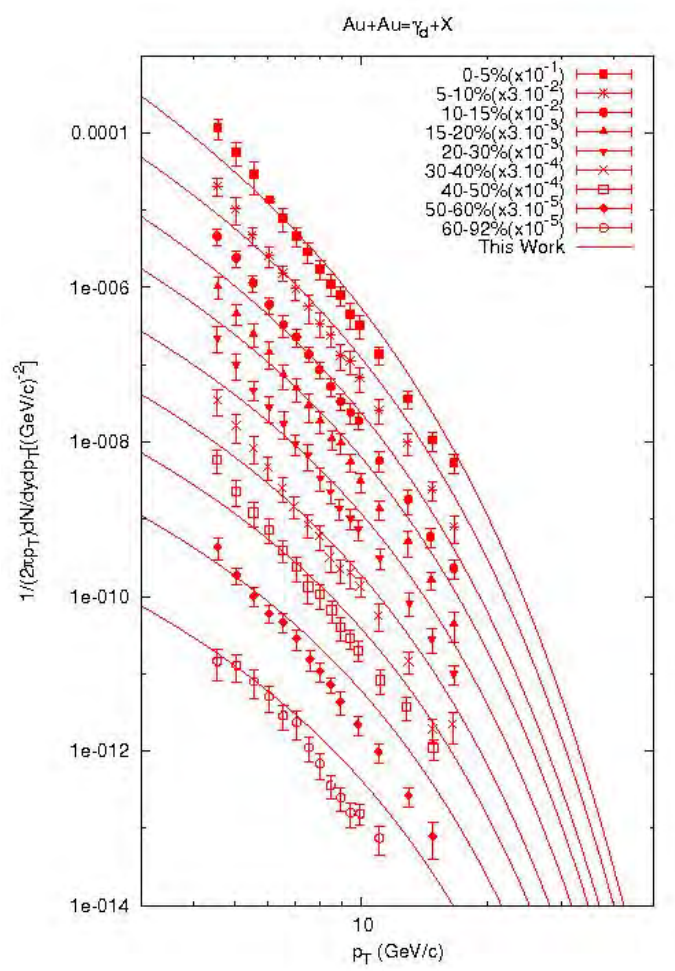

Fig. 2: Plot of the invariant cross-sections for direct photon production for different centralities in gold-gold collisions at $\sqrt{s}_{N N}=200 \mathrm{GeV}$ as function of $p_{T}$. The data points are from (Adare et al., 2012). The solid lines show the SCM-based results

The second term in the right hand side of the equation (1), $1 / p_{T}^{N_{R}}$, the constituent rearrangement term arises out of the partonic rearrangements inside the proton. These rearrangements mean undesirable loss 
of energy, in so far as the production mechanism is concerned. The choice of $N_{R}$ would depend on the following factors: (i) the specificities of the interacting projectile and target, (ii) the particularities of the secondaries emitted from a specific hadronic or nuclear interaction and (iii) the magnitudes of the momentum transfers and of a phase factor (with a maximum value of unity) in the rearrangement process in any collision. Collecting and combining all these relation may be given by (Guptaroy et al., 2008).

$$
N_{R}=4<N_{\text {part }}>^{1 / 3} \theta,
$$

where $<N_{\text {part }}>$ denotes the average number of participating nucleons and $\theta$ values are the constants of proportionality and they are to be obtained phenomenologically from the fits to the data-points.

The set of relations to be used for evaluating the parameters $a_{\gamma_{d}}$ and $\Delta^{\gamma_{d}}$ of equation (1) are given below;

$$
a_{\gamma_{d}}=C_{\gamma_{d}} \frac{3}{2 \pi} \frac{\left(A \sigma_{B}+B \sigma_{A}\right)}{\sigma_{A B}} \frac{1}{1+a^{\prime}\left(A^{1 / 3}+B^{1 / 3}\right)}\left(\frac{1000}{s}\right)^{1 / 5}
$$

and

$$
\Delta^{\gamma_{d}} \cong \xi s^{-0.3}
$$

$C_{r d}$ of equation (3) is a normalization factor arising out for direct photon production in $p+p$ collisions at different energies.

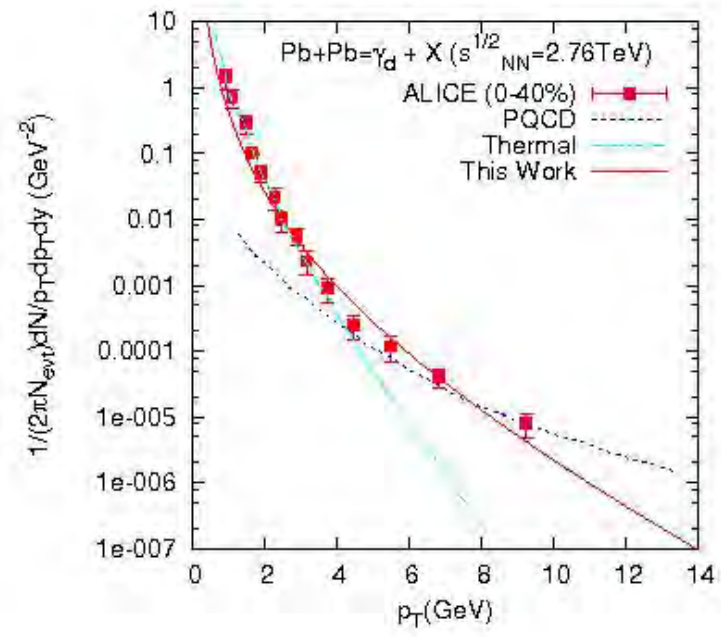

Fig. 3: Plot of the invariant yields for direct photon production in lead-lead collisions at $\sqrt{s}_{N N}=2.76 \mathrm{TeV}$ as function of $p_{T}$. The data points are from (Wilde et al., 2013). The solid line shows the SCM-based results while the dotted and dashed lines show the PQCD and Thermal approaches respectively (Nayak and Sinha, 2013)

The third factor of equation (3) gives a measure of the number of wounded nucleons i.e. the probable number of participants, wherein $A \sigma_{B}$ gives the probability cross-section of collision with ' $B$ ' nucleus (target), had all the nucleons of $A$ suffered collisions with $B$-target. $B \sigma_{A}$ has just the same physical meaning, 
with $A$ and $B$ replaced. Furthermore, $\sigma_{A}$ is the nucleon(proton)-nucleus(A) interaction cross section, $\sigma_{B}$ is the inelastic nucleon(proton)-nucleus(B) reaction cross section and $\sigma_{A B}$ is the inelastic $A B$ cross section for the collision of nucleus $A$ and nucleus $B$.

Besides, in expression (3), the fourth term is a physical factor related with energy degradation of the secondaries due to multiple collision effects. The parameter $a^{\prime}$ occurring in eqn.(3) above is a measure of the fraction of the nucleons that suffer energy loss. The maximum value of $a^{\prime}$ is unity, while all the nucleons suffer energy loss. This $a^{\prime}$ parameter is usually to be chosen (Wong, 1994), depending on the centrality of the collisions and the nature of the secondaries.

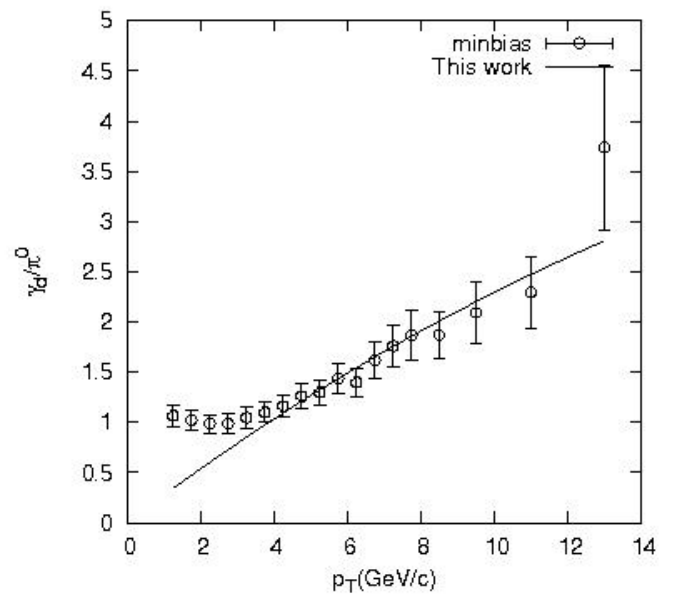

A

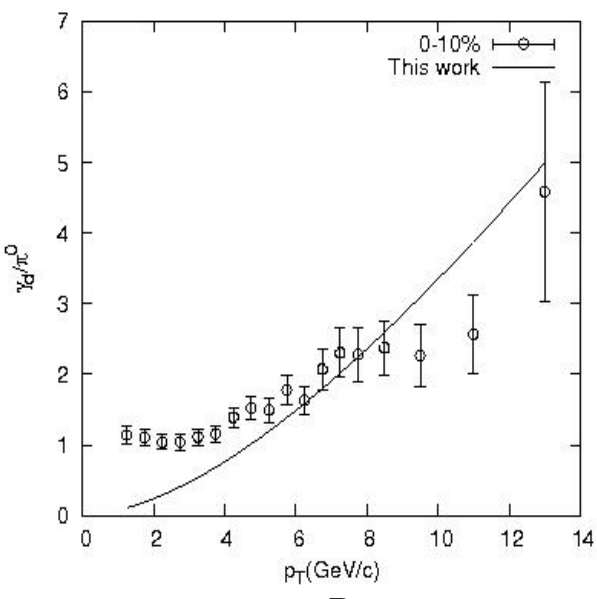

B

Fig. 4: The ratio of the direct photon to the neutral pion (as function of $p_{T}$ ) production in (A)Min. Bias and (B) 0-10\% Au + Au collisions at $\sqrt{s_{N N}}=200 \mathrm{GeV}$ are shown here. Data are taken from Ref.(PHENIX data, 2005). The solid lines show the SCM-based theoretical results

The " $a_{\gamma_{d}}$ " factor in the expression (1) accommodates a wide range of variation because of the existence of the large differences in the normalizations of the direct photon cross-sections for different types of interactions.

It is clear from expression (4), that the parameter $\Delta^{\gamma_{d}}$ has a particular energy dependence given in a specific form with $\xi$ as a numerical factor arising out of the proportionality constant and is treated here as a constant at a definite energy. For example, the value of $\xi$ for direct photon production is taken here to be 10.33 at $\sqrt{s_{N N}}=200 \mathrm{GeV}$.

\section{Results and Discussions}

In Fig. 1, we have drawn the solid lines depicting the model-based results for $p+p$ and $d+A u$ interactions against the experimental background (Adare et al., 2013). Moreover, in that figure, the dotted line represents 
NLO pQCD-based results (Adare et al., 2013). A point, in this context, needs to be addressed here. The disagreement observed in the low- $p_{T}$ by the SCM is due to the power law form of constituent rearrangement factor $1 / p_{T}^{N_{R}}$. The dominance of this power law form disturbs the agreement between data and model-based calculations in "soft" values. Whereas, for the case of NLO pQCD, the non-leading order-term in pQCD is responsible for better agreement of low- $p_{T}$ data as well as the high- $p_{T}$ data.

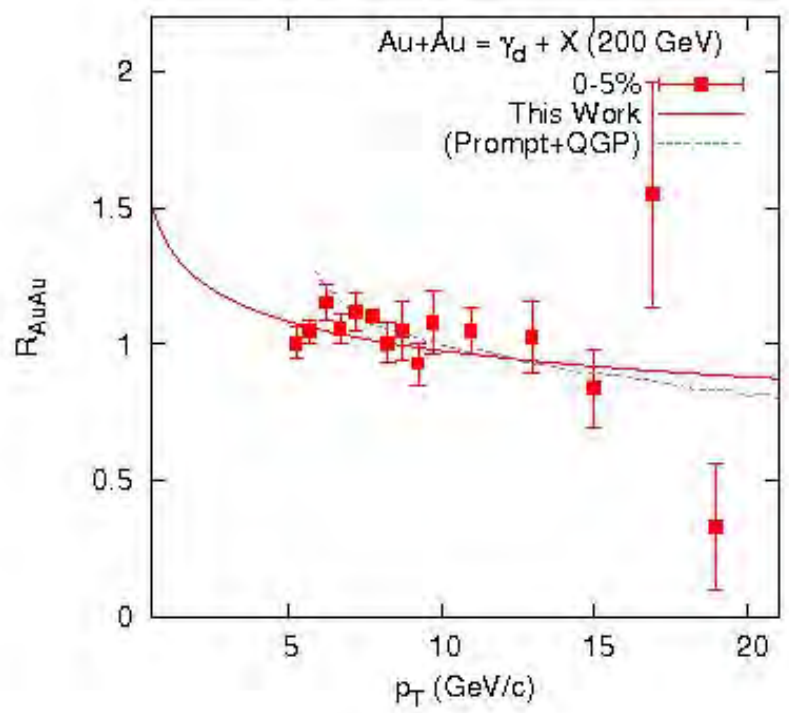

Fig. 5: Plot of $R_{A u A u}$ (as defined in the text) vs. $p_{T}$-values. Data are taken from (Adare et al., 2012). The solid line in the figure shows the SCM-based theoretical results. The other line is from the Ref. (Adare et al., 2012)

In a similar fashion, the invariant yields of direct photon in $A u+A u$ collision at RHIC energy $\sqrt{s}_{N N}=$ $200 \mathrm{GeV}$ for different centralities have been plotted in Fig.2. Data are taken from (Adare et al., 2012). The solid lines in the figure show SCM-based results.

In Fig. 3, we have plotted the invariant yields for $\mathrm{Pb}+\mathrm{Pb}$ collision at energy $\sqrt{s}_{N N}=2.76 \mathrm{TeV}$ at LHC. Data are taken from Ref.(Wilde et al., 2013). The solid line in the figure represents the SCM-based result while the dotted and dashed lines show the pQCD and thermal approaches respectively (Nayak and Sinha, 2013).

The direct photon to neutral pion ratio has been treated as an important physical observable. The difficulty of direct photon measurement arises mainly from the background comprising the decay of $\pi^{0}$ and $\eta$-mesons after freeze-out of the collision systems. The $\gamma_{d} / \pi^{0}$ ratio, in regard to direct photon measurement, can provide us a measure of the difficulty of the experimental environment for making direct photon measurement. In Fig. 4 we have plotted $\gamma_{d} / \pi^{0}$ ratio vs. $p_{T}$ for min bias and 0-10\% centrality in $A u+A u$ 
collision at RHIC energy $\sqrt{s}_{N N}=200 \mathrm{GeV}$. The solid line shows the SCM-based results. Data are taken from the Ref.(PHENIX data, 2005).

There is yet another very important observable called nuclear modification factor (NMF) $R_{A B}$ which for production of the direct photon is defined (Isobe et al., 2007) by

$$
R_{A B}\left(p_{T}\right)=\frac{d^{2} N^{A B} / d p_{T} d \eta}{<T_{A B}(b)>d^{2} \sigma^{p p} / d p_{T} d \eta}
$$

In Fig. 5, we have shown $R_{A u A u}$ vs. $p_{T}$ for $A u+A u$ collision. The solid line in the figure shows the SCM-based results against the experimentally measured results (Adare et al., 2012) given by filled squares with error bars. The dotted line in the fig.5 represents 'Prompt+QGP' approach (Adare et al., 2012).

\section{Final Remarks}

The main conclusions of this work are: (i) The model proposed here for the production of direct photon comprises some well-measured coupling terms of $\rho-\omega-\pi$ and $\omega-\gamma$ and some other standard particle physics considerations. (ii) Selected comparisons of our model-based results reveal neither sharp disagreement with any of the other models which are generally of standard model varieties nor very good agreement with either of them. (iii) The issue of direct photon yields could be tackled even by such non-gluonization physics-approach.

\section{References}

1. Adare A et al. (PHENIX Collaboration) (2012) Measurement of Direct Photons in Au+Au Collisions at $\sqrt{s_{N N}}$ = $200 \mathrm{GeV}$ Phys Rev Lett 109152302

2. Adare A et al. (PHENIX Collaboration) (2013) Direct photon production in d+Au collisions at $\sqrt{s_{N N}}=200$ GeV Phys Rev C87 054907

3. Gorenstein MI, Kostyuk AP, Stöcker H and Greiner W (2002) $J / \Psi$ suppression and enhancement in $\mathrm{Au}+\mathrm{Au}$ collisions at the BNL RHIC Phys Lett B524 265-272

4. Guptaroy P and Guptaroy S (2013) Direct Photon Production at RHIC and LHC-energies: Measured Data Versus a Model Chin Phys Lett 30062502

5. Guptaroy P, Sau G, Biswas SK and Bhattacharyya S (2008) On Production of Direct Photons and Neutral Pions in RHIC Experiments at $\sqrt{s_{N N}}=200 \mathrm{GeV}$ Mod Phys Lett A23 1031-1046

6. Guptaroy P, De B and Bhattacharyya S (2003a) On Direct Photon Production in $\mathrm{Pb}+\mathrm{Pb}$ and $\mathrm{Au}+\mathrm{Au}$ Collisions Heavy Ion Physics 17 167-177

7. Guptaroy P, De B and Bhattacharyya S (2003b) Direct photon Production in High Energy Hadronic and Nuclear Collisions: Measured Data Versus a Model Int. J Mod Phys A18 5047-5068 and the references therein

8. Isobe T for PHENIX Collaboration (2007) Systematic study of high- $p_{T}$ direct photon production with the PHENIX experiment at RHIC J Phys G34 S1015-S1018 
9. Nayak J and Sinha B (2013) Electromagnetic signals from Au+Au collisions at RHIC energy, $\sqrt{s_{N N}}=200$ $\mathrm{GeV}$ and $\mathrm{Pb}+\mathrm{Pb}$ collisions at LHC energy $\sqrt{s_{N N}}=2.76 \mathrm{TeV}$, Phys Lett $\mathrm{B} 719$ 110-115

10. PHENIX data (2005) http://www.phenix.bnl.gov/phenix/WWW/info/data/ppg042 data.html

11. Wilde $\mathrm{M}$ for the ALICE Collaboration (2013) Measurement of Direct Photons in pp and $\mathrm{Pb}-\mathrm{Pb}$ Collisions with ALICE, Nucl Phys A904-905 573c-576c

12. Wong CY (1994) Introduction to High-Energy Heavy Ion Collisions (Singapore:World Scientific). 